\title{
The effectiveness of transcranial direct current stimulation as an add-on modality to graded motor imagery for treatment of complex regional pain syndrome: A randomized proof of concept study
}

Émilie Lagueux, OT, PhD ${ }^{\text {a,b }}$, Michaël Bernier, MSc ${ }^{\text {a,c }}$, Patricia Bourgault, RN, PhD a,d, Kevin Whittingstall, $\mathrm{PhD}{ }^{\mathrm{a}, \mathrm{c}}$, Catherine Mercier, OT, $\mathrm{PhD}{ }^{\text {e,f }}$, Guillaume Léonard PT, $\mathrm{PhD}^{\text {b,h }}$, Sarah Laroche, OT ${ }^{\mathrm{b}}$, Yannick Tousignant-Laflamme, PT, $\mathrm{PhD}$

a. Research Center of the Centre Hospitalier Universitaire de Sherbrooke (CRCHUS), Québec, Canada

b. Faculty of Medicine and Health Sciences, School of Rehabilitation, Université de Sherbrooke, Québec, Canada

c. Faculty of Medicine and Health Sciences, Department of nuclear medicine and radiobiology, Université de Sherbrooke, Québec, Canada

d. Faculty of Medicine and Health Sciences, School of Nursing, Université de Sherbrooke, Québec, Canada

e. Faculty of Medicine, Department of Rehabilitation, Université Laval, Québec, Canada

f. Center for Interdisciplinary Research in Rehabilitation and Social Integration (CIRRIS), Québec, Canada

h. Research Center on Aging, CSSS-IUGS, Sherbrooke, Québec, Canada

* Corresponding author. Faculty of Medicine and Health Sciences, School of Rehabilitation, Université de Sherbrooke, 3001, 12 Avenue Nord, Z7, Sherbrooke, Québec, Canada, J1H 5N4

E-mail address: Yannick.Tousignant-Laflamme@USherbrooke.ca

Telephone: (819) 821-8000 extension 72912

Fax: (819) 820-6468

\section{Funding}

This study was supported by grants from the Canadian Pain Society (CPS), the Quebec Pain Research Network (QPRN), as well as the Inflammation and Pain Axis and the Faculty of Medicine and Health Sciences from the Université de Sherbrooke. CM and GL were supported by salary awards from the Fonds de recherche Québec - Santé (FRQS). KW holds a Canada Research Chairs (CRC). YTL is a supported member of the Quebec Pain Research Network. 


\begin{abstract}
Background: The efficacy of graded motor imagery (GMI) for the management of complex regional pain syndrome (CRPS) is supported by evidence, but its treatment effect remains generally modest. Transcranial direct current stimulation (tDCS) has been advocated as an adjunct intervention to enhance the effect of motor imagery approaches in pain populations.

Objective: The purpose of this study was to investigate the effectiveness of GMI + active tDCS compared to the GMI + sham tDCS in the treatment of CRPS type I.

Methods: 22 patients ( $\mathrm{n}=11 /$ group) were randomly assigned to the experimental (GMI + tDCS) or placebo (GMI + sham tDCS) group. GMI treatments lasted 6 weeks; anodal tDCS was applied over the motor cortex for 5 consecutive days during the first 2 weeks and once a week thereafter. Changes in pain perception, quality of life, kinesiophobia, pain catastrophizing, anxiety and mood were monitored after 6 weeks of treatment (T1) and 1-month post treatment (T2).

Results: GMI + tDCS induced no statistically significant reduction in pain compared to GMI + sham tDCS. Although we observed significant group differences in kinesiophobia $(\mathrm{p}=0.012)$, pain catastrophizing $(\mathrm{p}=0.049)$ and anxiety $(\mathrm{p}=0.046)$ at $\mathrm{T} 1$, these improvements were not maintained at $\mathrm{T} 2$ and did not reached a clinically significant difference.

Discussion: We found no added value of tDCS combined with GMI treatments for reducing pain in patients with chronic CRPS. However, given that GMI + sham tDCS induced no significant change, further studies comparing GMI + tDCS and tDCS alone are needed to further document tDCS's effect in CRPS.
\end{abstract}

Keywords: Complex regional pain syndrome; Non-invasive brain stimulation; Graded motor imagery; Pain management.

\title{
The authors declare no conflict of interest
}

Copyright (C) 2017 Wolters Kluwer Health, Inc. Unauthorized reproduction of the article is prohibited. 
Abbreviations: BDI-II = Beck Depression Inventory; BPI-sf = Brief Pain Inventory short form; CRPS = Complex Regional Pain Syndrome; GMI = Graded Motor Imagery; IMMPACT = Initiative on Methods, Measurement and Pain Assessment in Clinical Trials; M1 = Primary Motor Cortex MCID = Minimum Clinically Important Difference; MDC $=$ Minimal Detectable Change; NRS = numeric rating scales; PCS = Pain Catastrophizing Scale; SF12v2 = SF-12 short form; SF-12 MCS = SF-12 mental component summary; SF-12 physical component summary $($ SF-12 PCS $) ;$ STAI = State-Trait Anxiety Inventory; tDCS = transcranial Direct Current Stimulation; TSK = Tampa Scale of Kinesiophobia.

\section{Introduction}

Patients with complex regional pain syndrome (CRPS) present with multiple symptoms, including pain, sensory, motor, and trophic changes, associated with an impairment of autonomic control of the affected limb. ${ }^{1}$ The evolution of CRPS symptoms shows high inter-individual variability and symptoms can be classified in two phases - acute and chronic. ${ }^{2}$ While the transition from acute to chronic phase is not precisely determined, it is generally considered to occur within three to six months after the onset of the disease. ${ }^{2}$ The pathophysiology of CRPS is debated and there are possibly multiple mechanisms, including inflammation as well as changes in sympathetic, peripheral and central nervous systems. ${ }^{3,4}$ Research using functional imaging and electroencephalogram mapping suggests that CRPS may be a result of maladaptive plastic changes to the central nervous system. ${ }^{5,6}$ These studies demonstrated increased overlap between the representations of different body parts, potentially related a decrease in intracortical inhibition, ${ }^{7}$ topographical shrinkage in cortical activation, and reduction in the size of the somatosensory homunculus (i.e. reduced face-to-hand distance). ${ }^{6,8,9}$ Hence, modulating cortical activity might be a noteworthy therapeutic avenue for individuals suffering from CRPS. ${ }^{9-11}$ 
Interestingly, studies using graded motor imagery (GMI) in the treatment of CRPS reported positive results, and it has been suggested that the normalization of cortical reorganization seen in these patients might be the underlying mechanism to patient recovery. ${ }^{4,12,13}$ GMI aims to activate cortical networks involved in sensorimotor processing in three progressive phases: 1) a left-right limb discrimination task (recognition), 2) imagined movements of the affected limb, and 3) mirror therapy. ${ }^{14,15}$ The most recent practice guidelines for the treatment of CRPS state that GMI should be used as a first line treatment, ${ }^{3}$ as evidence shows that a 6-week GMI program offers better reduction in pain and improvements in functional gains when compared to conventional rehabilitation program. ${ }^{14,16,17}$ However, despite these positive results, the treatment effect size remains modest and for up to a third of the patients, the symptoms of CRPS often persist for years. ${ }^{13,14,16-18}$ Moreover, considering that a recent Cochrane review rated the quality of evidence for GMI as low, ${ }^{19}$ it appears appropriate to investigate new therapeutic approaches that could enhance current non-pharmacological treatments for CRPS. As suggested by the most recent practice guidelines, motor cortex stimulation, through transcranial Direct Current Stimulation (tDCS), is as an experimental therapy that could be instated in parallel to GMI. ${ }^{3}$ Other studies have shown that the combination of tDCS with a non-pharmacological treatment (e.g. visual illusion of walking) in the treatment of neuropathic pain related to spinal cord injury resulted in a mean reduction of $29.7 \%$ in pain intensity compared to $11.0 \%$ to visual illusion combined with placebo tDCS. ${ }^{20}$ Similar results were also observed in a case study involving a patient with CRPS type I, which found beneficial effects of tDCS on chronic pain and quality of life after the addition of tDCS to sensorimotor rehabilitation. ${ }^{21}$ To our knowledge, outside these discussed there are no other studies that have explored tDCS effects' in CRPS patients. 
Given that a recent Cochrane review ${ }^{10}$ concluded that tDCS may not deliver important benefits in chronic pain, a combined approached is justified. Thus, the main goal of this study was to explore the effectiveness of anodal tDCS in the treatment of CRPS in addition to GMI on the severity of pain in patients with CRPS type I. We hypothesized that the combination of tDCS with GMI would lead to better outcomes than GMI alone.

\section{Materials and methods}

\section{Participants}

In accordance with the Declaration of Helsinki, the study was approved by the Ethics Review Boards of the Centre hospitalier universitaire de Sherbrooke and the Hôpital Charles-Le Moyne (Québec, Canada). This trial was also registered at ClinicalTrials.gov [NCT01960400]. All participants (22 selected out of 62 potential candidates) were patients recruited from the pain clinics of these two hospitals and all provided their written informed consent prior to the start of the experiment. Participants received no financial compensation for their participation. Eligible patients were (i) aged $\geq 18$ years old; (ii) had a medical diagnostic of CRPS type 1 confirmed by an anesthesiologist or a neurosurgeon based on Budapest Research Criteria for research ${ }^{3}$; (iii) did not have other chronic pain conditions, dyslexia, visual impairment, neurological, psychological or motor disorder; (iv) did not have any history of brain implants, severe head trauma or headaches; and finally (v) if the patient was taking pain medication, their pharmacological intervention had to be stable (unchanged dose) over the last 4 weeks prior to the beginning of the study. Furthermore, participants were required not to modify their pharmacological and/or non-pharmacological treatments throughout the 10 weeks of the trial (see Table 1 for overview of patient characteristics). 


\section{Experimental design}

A randomized parallel single blind design including two treatment options was used (GMI + active tDCS; GMI + sham tDCS). This design was selected to provide initial evidence regarding the effectiveness of the combined treatment for further large-scale studies. ${ }^{22}$ Since the prevalence of CRPS is low, sampling was non-probabilistic and patients consisted of a consecutive sample of all eligible patients. After the verification of the admissibility criteria, patients were randomly assigned to the experimental group (GMI + active tDCS) or the placebo group (GMI + sham tDCS) using a randomized block design, to balance groups in regards to the duration of symptoms. Hence, a list was made for randomization into blocks ( 2 X 2$)$, one block for treatment group and one block for duration of symptoms $(\leq 6$ months and $>6$ months since diagnosis of CRPS type 1). In order to avoid a potential concealment bias, the randomization sequence was concealed from the investigators, where only an independent research agent held the allocation list. The research team did not know the exact size of the blocks until the experiment was completed.

\section{Graded motor imagery (GMI) intervention}

Home exercise: The GMI intervention was performed at home using the Recognise ${ }^{\mathrm{TM}}$ Flash Cards set, consisting of 25 left and 25 right matching images (upper limb or lower limb) and a mirror box (NOIgroup, Adelaide City West, South Australia). The participants performed the GMI therapy at home, 10 minutes per session, 3 times per day, 6 times a week, for 6 weeks. ${ }^{23}$ The description and settings of the GMI process are detailed in appendix (see Appendix A, Supplemental Digital Content 1, http://links.lww.com/CJP/A446 ). Before the beginning of each phase, the clinician carefully explained the GMI program to the participant. To further enhance standardization, a guide explaining the treatment parameters was given to all participants. 
Furthermore, at each visit, the GMI program was reviewed with the participant and, if needed, the clinician provided the necessary adjustments to follow the standardized application of the program.

\section{Transcranial direct current stimulation (tDCS) intervention}

Interventions in the experimental (clinical) settings: tDCS was delivered according to the method described by Fregni et al. (2006) ${ }^{24}$ and in accordance with the safety guidelines related to tDCS application. ${ }^{25}$ Direct current was delivered using a battery-driven constant current stimulator (DC-Stimulator NeuroConn, Germany) coupled to saline-soaked $(0.9 \% \mathrm{NaCl})$ sponge electrodes (5 X $7 \mathrm{~cm}$ ). Anodal stimulation was delivered over M1; the anode was placed over C3 or C4 position in the 10/20 system) for the EEG electrode position, contralateral to the affected limb, and the cathode over the opposite supraorbital area (i.e. ipsilateral to the affected limb). ${ }^{25}$ The choice of stimulation sites and stimulation parameters was based on previous studies showing improvement of neuropathic pain conditions. ${ }^{20,27}$ A constant current of an intensity of $2 \mathrm{~mA}$ was applied for 20 minutes (30 seconds ramp up, 30 seconds ramp down), each day, for five consecutive days (Monday to Friday) during the first and the second weeks of GMI (see Fig. 1A). To help retain the potential effects of the neurostimulation, the tDCS was also applied simultaneously with GMI once a week (Mondays) during the two other phases until the end of the six weeks GMI program, for a total of 14 treatment sessions. For the group receiving the sham tDCS, the electrodes were placed in the same position as for the active stimulation using the same tDCS stimulator at same stimulation intensity $(2 \mathrm{~mA})$, but the device automatically turned off after 30 seconds of stimulation (placebo mode). This type of sham stimulation has been shown to reliably blind subjects. ${ }^{26}$ 


\section{Timing of GMI and tDCS interventions}

All combined GMI and tDCS intervention occurred in the experimental (clinical) setting. During weeks 1 and 2 (Monday to Friday), the first GMI treatment of the day was combined with tDCS; the other two prescribed GMI treatments for that day were performed at home. In addition, on Saturday or Sunday, participants realized the GMI program three times per day (a total of 10 treatments combined in laboratory and 26 treatments GMI at home). For weeks 3 to 6, GMI + tDCS treatments were done on Monday mornings, and all other daily GMI sessions were undertaken by the patient at home, for a total of 4 treatments combined in laboratory and $68 \mathrm{GMI}$ sessions at home (see Figure 1a).

Accordingly, when patients were present in the experimental setting, the tDCS was installed and started. After 8 minutes of stimulation (out of 20), they were asked to start performing the GMI exercises (see Figure 1b). This was based on the observations of Nitsche \& Paulus, who found that anodal stimulation of about 9 to 13 minutes leads to a more robust and long-lasting increase in cortical excitability. ${ }^{28}$

Fidelity of the intervention:

In order to ensure the fidelity of the application of the combined treatment within the three clinicians, and to ensure that the results obtained were dependent on the intervention and not on the clinician, specific strategies were put in place: 1) the production of an application guide - as recommended by Sidani \& Braden $^{29}$ - containing a detailed description of the combined interventions, the materials needed and the procedure to be followed by the research team members, 2) a priori standardization of clinicians and 3) individual follow-up of the intervention for each participant by the lead investigator (EL). 


\section{Outcome measures (dependent variables)}

The choice of the outcome measures was made in accordance with the Initiative on Methods, Measurement and Pain Assessment in Clinical Trials (IMMPACT) guidelines ${ }^{30}$ and included measures of pain severity and interference with function. All patients completed a selfadministered questionnaire to assess the clinical characteristics of pain. Measurements took place before (T0), after 6 weeks of treatment (T1) and 1 month after the end of the treatment (T2). The primary outcome measure was pain severity and measured by the Brief pain inventory shortform (BPI-sf). ${ }^{31}$ The BPI-sf includes four questions on pain levels, where subjects were asked to rate intensity on a scale of 0 (no pain) to 10 (worst possible pain) in regards to: 1) pain at its worst in the last 24 hours, 2) pain at its least in the last 24 hours, 3) average pain over the last 24 hours and 4) pain right now (present pain). The score of each subscale ranges from 0 to 10 for a total score of 40. The minimum clinically important difference (MCID) is an average score of $>2 / 10$ for each of the four subscales). ${ }^{32}$ The following secondary outcome measures were also assessed.

Impact of pain on daily function

The BPI-sf also had 7 questions measuring the impact of pain on daily function, including the impact on general activity, sleep, mood, enjoyment of life, walking ability, ability to work and perform daily tasks and relationships with other people. ${ }^{33}$ Subjects were asked to rate how their pain interfered with these items over the previous $24 \mathrm{~h}$ using a numerical rating scale (NRS) ranging from 0 (does not interfere) to 10 (completely interferes). The total score of this section of the BPI-sf ranges from 0 to 70 .

Quality of life 
The SF-12 short form (SF12v2) ${ }^{34}$ is a general health-related quality of life questionnaire that consists of 12 Likert scale questions derived from the original SF-36 questionnaire. ${ }^{35}$ It covers eight domains (i.e., physical functioning, role-physical, bodily pain, general health, vitality, social functioning, role-emotional and mental health). The scores on each of these domains are summarized into two scales: the SF-12 physical component summary (SF-12 PCS) and the SF12 mental component summary (SF-12 MCS). Scores for each summary scale, ranging from 0 to 100, were calculated with standard scoring algorithms and normalized using the US general population values [mean 50 (S.D. 10)], with lower scores indicating worse health-related quality of life. ${ }^{35}$ The MCID for the SF-12 PCS is $\geq 8.8 / 100$ and $\geq 9.3 / 100$ for the SF-12 MCS. ${ }^{36}$

\section{Kinesiophobia}

The Tampa Scale of kinesiophobia (TSK), ${ }^{37}$ a 17 -item self-report measure, was used to assess fear of movement and injury/(re)injury. The total score ranges from 17 to 68, with higher scores representing stronger fear-avoidance beliefs. ${ }^{38}$ The minimal detectable change (MDC) for the TSK is $>4.8 .^{39}$

\section{Pain Catastrophizing}

The Pain catastrophizing scale (PCS) ${ }^{40}$ was used to evaluate the feelings, thoughts, and emotions related to pain catastrophizing. It is a self-administered questionnaire consisting of 13 items included in three subscales. The total score ranges from 0 to 52 points, with higher scores representing stronger pain catastrophizing. ${ }^{40}$ The MDC for the PDC is $>9.1 .{ }^{39}$

\section{State Anxiety}

The State-Trait Anxiety Inventory (STAI) ${ }^{41}$ is used to assess the state of anxiety at the moment. ${ }^{42}$ The total score ranges from 20 to 80 , where higher total score is, higher is the anxiety about an event. 


\section{Symptoms of depression}

The Beck Depression Inventory (BDI-II), ${ }^{43}$ which includes 21 statements, was used to measure symptoms and attitudes specific to depression. ${ }^{44}$ Each statement is graded from 0 to 3 , and higher total score indicates more severe depressive symptoms. The MCID is a $\geq 17.5 \%$ reduction from baseline $(\geq 11 / 63){ }^{45}$

Measures of compliance, adverse effects and blinding

The completion of a daily diary at home by all the patients throughout the study enabled us to monitor the implementation of the GMI program as well as compliance. Patient blinding was assessed at the end of the trial by asking each patient to guess which treatment they believed to have received. Throughout and after all the GMI or tDCS treatments, patients were also asked to note and report any side effects they experienced.

\section{Statistical analysis}

Sample size estimates were based on the results of the study of Soler et al. (2010), ${ }^{20}$ which aimed to evaluate the efficacy of tDCS combined with another treatment modality, the visual illusion of walking. In their study, the severity of pain, assessed using a numerical scale from 0 10 , had an average reduction of $29.7 \pm 5.0 \%$ for the tDCS + visual illusion of walking group, compared to an average reduction of $11.0 \pm 14.0 \%$ for the sham tDCS + visual illusion of walking after treatment. To consider that there is a significant difference between both groups, pondering that the average percentage of pain relief would be $29.7 \%$ for the experimental group and $11.0 \%$ for the placebo group, we estimated a mean difference of $\sim 20 \%$ between groups, with a standard deviation of $14.0 \%(\alpha=0.05$ and $\beta=1$ to 0.80$)$. Hence, the sample size of each group was estimated at 10 participants. Considering a dropout rate of $10 \%$, a total of 11 participants per group were recruited. 
At T0, the normality of the data (sociodemographic characteristics and dependent variables) was first tested using the Kolmogorov-Smirnov test. According to the results, either parametric tests (Student's T tests; normal distribution) or non-parametric tests (Wilcoxon signed-rank tests; asymmetric distribution) were performed. For categorical data, the chi-square tests or Fisher exact tests (if frequency of a cell of the crosstab was five or less) were used to compare outcomes between the two groups.

To compare the effectiveness of interventions (between-group differences), a mixed-model ANOVA (time $\mathrm{X}$ group interaction) was used. Due to a difference in total duration of each measurement time $(\mathrm{T} 0$ - T1 = six weeks; $\mathrm{T} 1$ - T2 = four weeks), a simple contrast (versus $\mathrm{T} 0)$ and non-polynomial analysis was selected from the IBM SPSS software. Age, as a covariate was included in the model, since it was found to be significantly different between both groups in order to obtain an adjusted p-value. However, for all the continuous dependent variables, age did not significantly impact the results and therefore were not retained. To compare the percent of change of each individual against the recognized MCID, defined as the statistically determined smallest change in a treatment outcome to be identified as important for the patients, ${ }^{46}$ we used the Fisher's exact test for between groups comparison at T1 and T2. All statistical analyzes were performed using IBM SPSS Statistics 22.0 version of Mac OS and Graph Pad Software (2015) (http://www.graphpad.com/quickcalcs/).

\section{Results}

\section{Descriptive data}

All subjects were enrolled between May 2012 and June 2014. Figure 2 shows the CONSORT flowchart for our study. Twenty-two patients, all meeting the admissibility criteria, were included. 
At baseline (T0), sociodemographic and clinical characteristics of both groups did not differ significantly (see Table 2), except for the mean age in the experimental group (40.91 years \pm 8.83) versus for the placebo group $(52.73$ years \pm 10.48$)(\mathrm{p}=0.029)$. The mean duration of symptoms did not differ between both groups (experimental group: 36.27 months \pm 25.62 ; placebo group: 36.55 months \pm 25.79 ) (see Table 1).

Primary outcome measure: Pain severity (BPI-sf)

Overall, change in pain severity (BPI-sf) did not significantly differ between groups, where only a tendency was observed $(\mathrm{p}=0.065)$ (see Table 2$)$. The only Group X Time interaction was found in the subscale measuring pain severity (present pain $\mathrm{p}=0.046$ ), which was not maintained at the one-month post-intervention (T2) (see Figure 3). Also, no difference in the proportion of patients who reached the MCID threshold was found between both groups at T1 and at T2, even for the present pain subscale, where a $42 \%$ reduction in present pain intensity was observed at $\mathrm{T} 1$ for the experimental group.

\section{Secondary outcome measures}

Complete results for the secondary variables are also presented in Table 2. We found no significant between group differences (time $\mathrm{X}$ group interaction) at $\mathrm{T} 1$ and $\mathrm{T} 2$ regarding the impact of pain on global physical functioning, on the physical and mental health quality of life and on depressive symptoms. No difference in the proportion of patients who reached the MCID threshold was found between both groups for either variable, at T1 and at T2. For the fear of movement, pain catastrophizing and state anxiety, the analysis revealed a statistically significant time $\mathrm{X}$ group interaction at T1 only, which showed lower scores for the experimental group. However, these were not maintained at T2. Yet, considering that the group differences for the 
first two variables are less than the known MDC, it certainly did not reach a clinically significant level and has to be interpreted with caution.

\section{Assessment of compliance and blinding}

In regards to compliance, we noted from the daily diary that all participants reported completing their GMI sessions 3 times per day, 6 times a week, for 6 weeks. Finally, all patients completed the 14 treatments of tDCS in the laboratory setting.

At the end of the treatments (T1), participants were asked in which treatment group they believed they were allocated (experimental or placebo). Thirteen participants $(59.1 \%)$ responded correctly. Nine participants $(81.8 \%)$ from the experimental group compared with seven participants $(63.6 \%)$ from the placebo group responded that they had received the active tDCS treatment regimen. No statistically significant difference was found between groups in the assessment of blinding $(\mathrm{p}=0.635)$.

\section{Adverse effects of treatment}

Regarding the undesirable effects of the GMI treatment, three participants reported that specific images had generated a temporary increase in pain ( $~ 30$ minutes), mainly during the phase 2 of the protocol. Two strategies were proposed to these participants: to remove the images that provoked pain and to reduce the time of subsequent sessions (before the onset of pain). Only one participant from the experimental group reported minor fasciculation during the exercises in Phase 3. As a strategy to avoid this discomfort, the participant was invited to perform his exercises more slowly, by granting short breaks of a few seconds. Regarding the undesirable effects of the tDCS, three participants from the experimental group and two participants from the placebo group complained of minor headaches after tDCS, especially during the second week of Phase 1. In all cases, the headaches disappeared within two or three hours following the tDCS 
session. These participants reported taking acetaminophen after their tDCS session, which had a beneficial effect on their headaches. Only one participant from the experimental group reported a slight reddening and itching on his head (around the location of the anode) during the second week of treatment. Two participants from the experimental group and one participant of the placebo group reported feeling more tired than usual on a number of occasions after their tDCS treatment. Finally, four participants (18.2\%) declared having been indisposed by a sting/burning sensation under the electrodes during the first minute of active tDCS. Adding saline water $(\sim 5$ $\mathrm{ml}$ per electrode) and applying a slight pressure on the electrodes immediately reduced this discomfort. No tDCS sessions were interrupted due to undesirable or adverse effects.

\section{Discussion}

The main objective of this study was to produce preliminary evidence regarding the therapeutic effectiveness of the combination of GMI and tDCS for the management of CRPS. In regards to our main outcome measure (pain severity), we found no statistically significant group differences immediately and 1 month after the 6 weeks of treatment. Yet, considering that a tendency was observed $(\mathrm{p}=0.06)$ and that for one subscale of our main dependent variable a positive outcome was found, our results may suggest that this treatment regimen could induce positive outcomes on a very short-term perspective. Considering this tendency, a larger sample-size, thus greater power, could have lead to a true and significant effect. However, although stronger than GMI alone, these observations lead us to believe that, in our sample, the analgesic effects of GMI + tDCS are modest at the very and also very short-lived. As for group differences in regards to kinesiophobia, pain catastrophizing and state anxiety following 6 weeks of GMI + active tDCS, they need to be interpreted with caution since none reached a clinically significant difference. 
Short-term benefits of GMI + active tDCS

The combination GMI + active tDCS (experimental group) resulted in a $42 \%$ reduction of present pain intensity scores at $\mathrm{T} 1$; considering this change is greater than $\sim 30 \%$, it represents a clinically important difference. ${ }^{47}$ In comparison, this positive short-term effect is somewhat comparable to the $29.7 \%$ pain reduction obtained by Soler et al. (2011) ${ }^{20}$ with a comparable treatment approach (tDCS + visual illusion of walking), but in a different neuropathic pain population. However, this pain reduction should be interpreted with caution, as the effect is dependent on a subscale of only one of our main variable. In fact, the lack of significant effect in our main variable (pain severity) appears to be more similar to reports of a recent Cochrane systematic review, which concludes that tDCS may not deliver statistically significant effects in individuals with chronic pain. ${ }^{10}$ However, since we observed no changes in the placebo group (sham tDCS) - pain reduction was only observed in the experimental group (active tDCS) - the specific effects of the tDCS could explain the greater analgesia in present pain observed at T1. Given our results, a third group, which would have only received the active tDCS without GMI, could have helped us to determine the indebted effect specific to the tDCS. However, this was not performed due to: 1) the explorative nature of the study (proof of concept), 2) the feasibility issues in terms of recruiting participants and 3) ethical reasons given that GMI is recommended in current CRPS practice guidelines, ${ }^{3}$ it would have been arguable to provide only a treatment which is supposed to be an add-on to our patients. The effect of tDCS on present pain management could be an avenue to explore further, as the effect of tDCS was only sustained in the short term.

Lack of pain reduction one month after treatment (T2)

Copyright (C) 2017 Wolters Kluwer Health, Inc. Unauthorized reproduction of the article is prohibited. 
For the management of pain, the most commonly used protocol consisted of 20-min anodal stimulation of M1 for five consecutive days, which may lead to significant analgesic after-effects lasting for 2-6 weeks. ${ }^{48}$ According to the same parameters, the application of 10 sessions of anodal tDCS should generate long-lasting analgesic effects, up to 60 days after the end of treatments. ${ }^{27}$ However, although this dosage was observed in addition to a weekly session until the end of GMI treatment, the results we obtained (GMI + tDCS) did not demonstrate a significant analgesic after-effect lasting one month post-intervention.

An element that could explain the overall lack of effect might be related to anticonvulsants that might have decreased the effects of anodal stimulation after a single session of tDCS. ${ }^{49}$ This lack of pain reduction observed might suggest that patients with anticonvulsants may respond less to this treatment. In fact, $73 \%$ and $64 \%$ of the patients took anticonvulsants in the experimental and placebo groups, respectively, which could potentially have lessened the tDCS effect. ${ }^{49,50}$ In order to increase the effect and the chances of longer-lasting pain relief, perhaps a more adequate and/or personalized pharmacological approach could help modulate treatment effects, ${ }^{51}$ a factor that was not manipulated in our study, but only partially controlled.

\section{Lack of treatment effect in the placebo group}

Although not the main focus of our study, the absence of a "no-treatment" group also forbids us to draw any clear conclusion to answer questions regarding if GMI alone (placebo group) was helpful, harmful or of no value. However, participants in the placebo group (GMI + sham tDCS) showed no positive treatment effect, which again is in discordance to the results of some previously published studies, who found a reduction in pain intensity after GMI alone. ${ }^{14,16,17}$ This lack of effect of the GMI observed in our study could partly be explained by two factors: 1) duration of CRPS symptoms: although the distribution of participants in the two groups 
(experimental and placebo) during randomization was successful, our "chronic" CRPS patients all had a much longer duration of symptoms ( 36 months) compared to the participants in Moseley’s previous publications ( 14 months). ${ }^{14,16,17}$ Studies from other groups also reported poor results with the use of GMI for chronic CRPS patients. For instance, McCabe et al. ${ }^{52}$ found an immediate analgesic effect using mirror therapy on acute-subacute CRPS patients, but reported no positive effect for chronic CRPS patients (> 14 months). The same observations were reported by Johnson et al., ${ }^{53}$ who even reported an increase in pain of $+37.5 \%$ during GMI for their chronic CRPS patients ( 23 months). Hence, it appears that GMI has equivocal results for patients with a longer duration of CRPS and clearly needs further investigation. 2) minor discrepancies between treatment regimen: the other factor that could explain the absence of treatment effect for the placebo group may be related to our GMI intervention protocol, which somewhat varied from the one used in Moseley's published clinical trial ${ }^{17}$ (see Appendice A for comparisons); our recommended frequency for GMI exercises was lower (3 exercise sessions per day) than Moseley's hourly recommendation. ${ }^{14,16,17}$ But many audited patients in these previous studies reported that complying with exercises every waking hour was not feasible for them (75\% compliance rate was reported). Our methodological choice regarding the frequency of the exercises was based on previous positive findings ${ }^{23}$ and the fact that this exercise regimen was more respectful of their daily routine and quality of life during the intervention. ${ }^{29,54}$ This methodological choice also probably contributed to the perfect compliance we observed for our two groups. Nevertheless, the lower frequency of the exercises remains a possible explanation of the lack of result of the GMI. 
Finally, another possible explanation for the lack of effect in our placebo group is related to the absence of progression of the training load. The use of software proposed by Moseley (2006) ${ }^{17}$, instead of Recognise ${ }^{\mathrm{TM}}$ Flash Cards, would have made it possible to graduate the requirements of the exercises. However, the parameters of use of the GMI are not entirely known and are difficult to reproduce. In short, further investigations are required to determine if the NOI group's Recognise $^{\mathrm{TM}}$ Flash Cards provide equivalent results to the use of the adaptive software.

Knowing that 1) the effectiveness of GMI has been demonstrated by three separate trials of GMI, all conducted by the same research team and 2) all were relatively small trials (13 to 37 participants), a high risk of bias may be present. ${ }^{19}$ In fact, another independent research team could not, as in our study, confirm the effectiveness of the GMI. ${ }^{53}$ Failure of the real-world implementation of GMI suggests that better understanding of both GMI methodology and its interaction with other treatment modality is required to ensure that GMI research results can be translated into clinical practice. Further studies would be necessary to determine the optimal frequency of exercises, while considering its pragmatic application in real-life.

\section{Potential bias related to the blinding procedures}

When we initially developed this protocol, we relied on the most robust available evidence to develop the tDCS protocol. It was then accepted that the use of a $2 \mathrm{~mA}$ stimulation presumed an effective blinding ${ }^{20,21,27}$. But recently, O'Connell et al. (2012) ${ }^{55}$ demonstrated that a $2 \mathrm{~mA}$ tDCS might not be associated with effective blinding when compared with the sham commonly using this procedure. Since our study had reported superior efficacy of active stimulation over sham and adequate participant blinding, assessor blinding was not possible. While we cannot predict the degree of influence that inadequate blinding may have had in our study, non-specific effects of interventions are known to be important in such clinical conditions. ${ }^{56,57}$ Further, there is 
evidence that incomplete blinding leads to exaggerated effects in clinical studies with subjective outcomes, ${ }^{58}$ and that placebo effects are larger with physical placebo interventions. ${ }^{57}$ Thus, the O'Connell study ${ }^{55}$ contends that clinical studies that have used $2 \mathrm{~mA}$ tDCS should be interpreted with renewed caution. Using intensities of $1 \mathrm{~mA}$ in future research may represent a more methodologically sound option, although it is plausible that reducing the intensity may reduce potential efficacy. Future studies of tDCS may benefit from other methods to optimise blinding, for example de facto masking in which the treatment is not blinded but both treatments are presented as the active one. ${ }^{59}$

\section{Future considerations}

Maybe better outcomes could be obtained if the treatment was individually adjusted according to the main neurophysiological process that explains the development and/or maintenance of CRPS symptoms, i.e. peripheral vs. spinal vs. central hypersensitivity or sympathetic neural system. ${ }^{1}$ Based on the principle that management of painful pathologies should be incorporated within a biopsychosocial framework, patients could be offered treatments grounded on the multiple different mechanisms driving pain and disability. Maybe, in our protocol, too much emphasis was directed towards nervous system hypersensitivity (GMI and tDCS mostly target supra-spinal hypersensitivity) and less on cognitive-emotional factors (i.e.: psychogenic drivers of pain or pain avoidance-behaviors) or environmental factors, such as attitudes of others or beliefs regarding work. It is possible that a patient-centered approach, based on how the person responds to treatment with the addition of other targeted therapeutic modalities, could induce a more efficient management of CRPS. ${ }^{60}$ Also, further investigations are required to elucidate the elements of GMI intervention that determine success and to identify the characteristics of 
responders. A better understanding of the relative weight of this each factor could allow to better personalizing the treatment protocol.

\section{Conclusions}

The current report was the first to investigate the added-value of tDCS to GMI in the treatment of CRPS type I. Our results show no significant added value of adding a series of tDCS in addition GMI treatments for reducing pain severity in patients with chronic CRPS type I.

\section{Acknowledgements}

We are grateful to all the patients who took part in the study, and to the members of the study team, Laurence Beaulieu-Boire, Gabrièle Beaudoin, and Catherine Houle from the Université de Sherbrooke. We also wish to thank Marie-Pier Garant for her helpful comments and suggestions for the statistical analyses. Finally, thanks are due to Dr. Étienne DeMédicis, Dr. Nicolas Patenaude, Dr. Frédéric Croteau, Dr. Jocelyn Pelletier, and Dr. Denis Roy who referred us potential participants.

\section{Figure legends:}

Figure 1: Experimental design

Figure 2: CONSORT flow chart

Figure 3: Progression of present pain intensity scores through time for both groups

Copyright (C) 2017 Wolters Kluwer Health, Inc. Unauthorized reproduction of the article is prohibited. 


\section{References}

1. Birklein F, O’Neill S, Schlereth T. Complex regional pain syndrome $\square$ : An optimistic perspective. Neurology. 2015;6(1):89-96.

2. Zyluk A, Puchalski P. Complex regional pain syndrome of the upper limb: a review. Neurol Neurochir Pol. 2014;48(3):200-205.

3. Harden R, Oaklander A, Burton A, et al. Complex regional pain syndrome: practical diagnostic and treatment guidelines, 4th edition. Pain Med. 2013;14(2):180-229.

4. Moseley G, Butler D, Beames T, Biles T. The Graded Motor Imagery Handbook. 1st ed. Adelaïde: Neuro orthopaedic Institute; 2012.

5. Bruehl S. Complex regional pain syndrome. Br Med J. 2015;29.

6. Marinus J, Moseley G, Birklein F, et al. Clinical features and pathophysiology of complex regional pain syndrome. Lancet Neurol. 2011;10:637-648.

7. Schwenkreis P, Janssen F, Rommel O, et al. Bilateral motor cortex disinhibition in complex regional pain syndrome (CRPS) type I of the hand. Neurology. 2003;61(4):515519.

8. Maihöfner C, Handwerker H, Neundorfer B, Birklein F. Patterns of cortical reorganization in complex regional pain syndrome. Neurology. 2003;61(1707-1715).

9. Pleger B, Tegenthoff M, Ragert P, et al. Sensorimotor retuning in complex regional pain syndrome parallels pain reduction. Ann Neurol. 2005;57(3):425-429.

10. O’Connell N, Wand B, Marston L, Spencer S, Desouza L. Non-invasive brain stimulation techniques for chronic pain. Cochrane Database Syst Rev. 2014.

11. Maihöfner C, Handwerker H, Neundorfer B, Birklein F. Cortical reorganization during recovery from complex regional pain syndrome. Neurology. 2004;63(4):693-701.

12. Prigane V, Stralka S. Graded motor imagery. J Hand Ther. 2011;24(2):164-168.

13. Walz A, Usichenko T, Moseley G, Lotze M. Graded motor imagery and the impact on pain processing in case of CRPS. Clin J Pain. 2013;29(3):276-279.

14. Moseley G. Graded motor imagery is effective for long-standing complex regional pain syndrome: A randomised controlled trial. Pain. 2004;108(1-2):192-198.

15. Ramachandran V, Altschulder E. The use of visual feedback, in particular mirror visual, in restoring brain function. Brain. 2009;132(7):1693-1710.

Copyright (C) 2017 Wolters Kluwer Health, Inc. Unauthorized reproduction of the article is prohibited. 
16. Moseley G. Is successful rehabilitation of complex regional pain syndrome due to sustained attention to the affected limb? A randomised clinical trial. Pain. 2005;114(12):54-61.

17. Moseley G. Graded motor imagery for pathologic pain $\square$ : A randomised controlled trial. Neurology. 2006;67(12):2129-2134.

18. Bowering K, O'Connell N, Tabor A, et al. The effects of graded motor imagery and its components on chronic pain $\square$ : a systematic review and metaanalysis. $J$ Pain. 2013;14(1):3-13.

19. Smart K, Wand B, O’Connell N. Physiotherapy for pain and disability in adults with complex regional pain syndrome (CRPS) types II and I. Cochrane Database Syst Rev. 2016.

20. Soler M, Kumru H, Pelayo R, et al. Effectiveness of transcranial direct current stimulation and visual illusion on neuropathic pain in spinal cord injury. Brain. 2010;133:2565-2577.

21. Schmid A, Hummel F, Birbaumer N. Pain reduction in a CRPS patient due to tDCS and sensorimotor training. A single case study. Clin Neurophysiol. 2011;122:S144.

22. Gewandter J, Dworkin RH, Turk D, et al. Research designs for proof-of-concept chronic pain clinical trials: IMMPACT recommendations. Pain. 2014;155(9):1683-1695.

23. Lagueux E, Charest J, Lefrançois-Caron E, et al. Modified graded motor imagery for complex regional pain syndrome type I of the upper extremity in the acute phase: a patient series. Int J Rehabil Res. 2012;35(2):138-145.

24. Fregni F, Boggio P, Lima M, et al. A sham controlled, phase II trial of transcranial direct current stimulation for the treatment of central pain in traumatic spinal cord injury. Pain. 2006;122(197-209).

25. DaSilva A, Volz M, Bikson M, Fregni F. Electrode positioning and montage in transcranial direct current stimulation. J Vis Exp. 2011;51.

26. Gandiga P, Hummel F, Cohen L. Transcranial DC stimulation (tDCS) $\square$ : a tool for double blind sham-controlled clinical studies in brain stimulation. Clin Neurophysiol. 2006;117(4):845-850.

27. Valle A, Roizenblatt S, Botte S, et al. Efficacy of anodal transcranial direct current stimulation (tDCS) for the treatment of fibromyalgia: results of a randomized, shamcontrolled longitudinal clinical trial. J Pain Manag. 2009;2(3):353-362.

Copyright (C) 2017 Wolters Kluwer Health, Inc. Unauthorized reproduction of the article is prohibited. 
28. Nitsche M, Paulus W. Sustained excitability elevations induced by transcranial DC motor cortex stimulation in humans. Neurology. 2001;57:1899-1901.

29. Sidani S, Braden C. Design, Evaluation and Translation of Nursing Interventions. Chichester, West Sussex: Wiley \& Sons Incorporated; 2011.

30. Dworkin RH, Turk DC, Farrar JT, et al. Core outcome measures for chronic pain clinical trials: IMMPACT recommendations. Pain. 2005;113(1):9-19.

31. Poundja J, Fikretoglu D, Guay S, Brunet A. Validation of the French version of the brief pain inventory in Canadian veterans suffering from traumatic stress. J Pain Symptom Manage. 2007;33(6):720-726.

32. Mease P, Spaeth M, Clauw D, et al. Estimation of minimum clinically important difference for pain in fibromyalgia. Arthritis Care Res (Hoboken). 2011;63(6):821-826.

33. Cleeland C, Ryan K. Pain assessment: global use of the Brief Pain Inventory. Ann Acad Med Singapore. 1994;23(2):129-138.

34. Leplège A, Mesbah M, Marquis P. Analyse préliminaire des propriétés psychométriques de la version française d'un questionnaire international de mesure de qualité de vie: le MOS SF-36 (versions 1.1). Rev Epidemiol Sante Publique. 1995;43(4):371-379.

35. Ware J, Kosinski M, Keller S. A 12-Item Short-Form Health Survey: construction of scales and preliminary tests of reliability and validity. Med Care. 1996;34(3).

36. Parker S, Mendenhall S, Shau D, et al. Determination of minimum clinically important difference in pain, disability, and quality of lifeafter extension of fusion for adjacentsegment disease. J Neurosurg Spine. 2012;16(1):61-67.

37. Kori S, Miller R, Todd D. Kinesophobia: A new view of chronic pain behaviour. Pain Manag. 1990;3:35-43.

38. Clark M, Kori S, Brockel J. Kinesiophobia and chronic pain: psychometrics characteristics and factor analysis of the Tampa Scale. Proc Am Soc Annu Meet. 1996;15(17).

39. George S, Valencia C, Beneciuk J. A psychometric investigation of fear-avoidance model measures in patients with chronic low back pain. J Orthop Sports Phys Ther. 2010;40(4):190-205.

40. Sullivan M, Bishop S, Pivik J. The Pain Catastrophizing Scale $\square$ : Development and validation. Psychol Assess. 1995;7:524-532.

Copyright (C) 2017 Wolters Kluwer Health, Inc. Unauthorized reproduction of the article is prohibited. 
41. Gauthier J, Bouchard S. Adaptation canadienne-française de la forme révisée du StateTrait Anxiety Inventory de Spielberg. Rev Can des Sci du Comport. 1993;25(559):578.

42. Spielberg C. Manual for the State-Trait Anxiety Inventory. Revised ed. Palo Alto, CA: Consulting Psychologist Press; 1983.

43. Bourque P, Beaudette D. Étude psychométrique du questionnaire de dépression de Beck auprès d'un échantillon d'étudiants universitaires francophones. Rev Can des Sci du Comport. 1982;14(3):211-218.

44. Beck A, Steer R, Brown G. BDI-II, Beck Depression Inventory $\square$ : Manual 2nd ed. San Antonio: The Psychological Corporation; 1996.

45. Button K, Kounali D, Thomas L, et al. Minimal clinically important difference on the Beck Depression Inventory--II according to the patient's perspective. Psychol Med. 2015;45(15):3269-3279.

46. Beaton D, Boers M, Wells G. Many faces of the minimal clinically important difference (MCID): a litterature review and directions for future research. Curr Opin Rheumatol. 2002;14(2):109-114.

47. Farrar J, Young J, LaMoreaux L, Werth J, Poole R. Clinical importance of changes in chronic pain intensity measured on an 11-point numerical pain rating scale. Pain. 2001;94(2):149-158.

48. Lefaucheur J-P, Antal A, Ayache SS, et al. Evidence-based guidelines on the therapeutic use of transcranial direct current stimulation (tDCS). Clin Neurophysiol. 2017;128(1):5692.

49. Liebetanz D, Nitsche M, Tergau F, Paulus W. Pharmacological approach to the mechanisms of transcranial DC-stimulation-induced after-effects of human motor cortex excitability. Brain. 2002;125(10):2238-2247.

50. Nitsche M, Fricke K, Henschke U, et al. Pharmacological modulation of cortical excitability shifts induced by transcranial direct current stimulation in humans. $J$ Physiol. 2003;553(1):293-301.

51. Brunoni A, Nitsche $\mathrm{M}$, Bolognini $\mathrm{N}$, et al. Clinical research with transcranial direct current stimulation (tDCS) $\square$ : challenges and future directions. Brain Stimul. 2012;5(3):175-195. 
52. McCabe C, Haigh R, Ring E, Halligan P, Wall P, Blake D. A controlled pilot study of the utility of mirror visual feedback in the treatment of complex regional pain syndrome. Rheumatology. 2003;42(1):97-101.

53. Johnson $\mathrm{S}$, Hall J, Barnett $\mathrm{S}$, et al. Using graded motor imagery for complex regional pain syndrome in clinical practice: failure to improve pain. Eur J Pain. 2012;16(4):550-561.

54. Brousselle A, Champagne F, Contandriopoulos A, Hartz Z. L'évluation $\square$ : Concepts et Méthodes. 2nd ed. Montréal, Qc: Les Presses de l’Université de Montréal; 2011.

55. O’Connell N, Cossar J, Martson L, et al. Rethinking clinical trials of transcranial direct current stimulation: participant and assessor blinding is inadequate at intensities of $2 \mathrm{~mA}$. PLoS One. 2012;7(10):e47514.

56. Brunoni A, Lopes M, Kaptchuk T, Fregni F. Placebo response of nonpharmacological and pharmacological trials in major depression: a systematic review and meta-analysis. PLoS One. 2009;4(3):e4824.

57. Hrobjartsson A, Gøtzsche P. Placebo interventions for all clinical conditions. Cochrane Database Syst Rev. 2010.

58. Wood L, Egger M, Gluud L, Juni P, Altman D. Empirical evidence of bias in treatment effect estimates in controlled trials with different interventions and outcomes: Metaepidemiological study. Br Med J, 2008;336(7644):601-605.

59. Berger V. De facto masking and other measures to prevent contamination. J Clin Epidemiol. 2012;65(11):1236-1237.

60. Gobel A. Current concepts in adult CRPS. Rev Pain. 2011;5(2):3-11. 
Table 1. demographic and clinical characteristics of the patients

\begin{tabular}{|c|c|c|c|c|c|c|}
\hline Gender & $\begin{array}{l}\text { Age } \\
\text { (years } \\
\text { ) }\end{array}$ & $\begin{array}{l}\text { Time } \\
\text { since Dx } \\
\text { CRPS } \\
\text { (months) }\end{array}$ & $\begin{array}{l}\text { Affected } \\
\text { limb }\end{array}$ & Initial event & $\begin{array}{l}\text { Pain level } \\
\text { baseline } \\
\text { mean } \\
(/ 10) \\
\end{array}$ & Pain medication \\
\hline \multicolumn{7}{|c|}{ Graded Motor Imagery (GMI) group + active transcranial Direct Current Stimulation (tDCS) } \\
\hline Female & 27 & 13 & Right hand & Sprain grade 2 & 5 & PGB, ACE, NSAID, ANT \\
\hline Male & 39 & 39 & Left foot & Sprain grade 2 & 5 & PGB, ANT \\
\hline Female & 51 & 19 & Right arm & Electrification & 8 & $\begin{array}{l}\text { PGB, NSAID, BZD, } \\
\text { ANT, BZD, CAL }\end{array}$ \\
\hline Female & 45 & 23 & Left hand & $\begin{array}{l}\text { Amputation distal } \\
\text { thumb }\end{array}$ & 3 & INN \\
\hline Female & 49 & $5^{* *}$ & Left arm & Wrist fracture & 4 & GBP, ACE \\
\hline Female & 53 & 127 & Right arm & Repetitive movement & 6 & PGB, TMD, ANT \\
\hline Female & 43 & $5^{* *}$ & Left foot & $\begin{array}{l}\text { Sprain and } \\
\text { microfracture }\end{array}$ & 2 & \\
\hline Female & 22 & 72 & Right hand & $\begin{array}{l}\text { Shoulder } \\
\text { hyperextension }\end{array}$ & 7 & GB, TMD \\
\hline Female & 37 & 48 & Right leg & Minor trauma & & \\
\hline Male & 31 & 23 & Left knee & Patella fracture & & GBP, BZD \\
\hline Male & 53 & 25 & Right foot & $\begin{array}{l}\text { Medial malleolus } \\
\text { fracture }\end{array}$ & 6 & BZD \\
\hline $\begin{array}{ll}\mathrm{F}: 8 & \mathrm{M}: 3\end{array}$ & $41 \pm 9$ & $36 \pm 26$ & $\begin{array}{l}R: 4(\mathrm{U}) ; 2(\mathrm{~L}) \\
\mathrm{L}: 2(\mathrm{U}) ; 3(\mathrm{~L})\end{array}$ & & $4.9 \pm 1.8$ & \\
\hline \multicolumn{7}{|c|}{ Graded Motor Imagery (GMI) group + sham transcranial Direct Current Stimulation (tDCS) } \\
\hline Female & 63 & 92 & Right arm & Wrist fracture & 7 & None \\
\hline Male & 58 & $3^{* *}$ & Left hand & Cyst ablation & 6 & GBP, PGB, COD \\
\hline Male & 46 & 36 & Left foot & Sprain and avulsion & 8 & $\begin{array}{l}\text { PGB, ANT, ACE, } \\
\text { NSAID, KTMt }\end{array}$ \\
\hline Female & 56 & 77 & Left arm & Wrist tendinitis & 8 & $\mathrm{BZD}, \mathrm{INN}, \mathrm{CBZ}, \mathrm{ANT}$ \\
\hline Female & 46 & 50 & Right hand & Minor trauma & 7 & $\mathrm{ANT}, \mathrm{CBZ}$ \\
\hline Female & 35 & 19 & Left foot & Crush and sprain & 6 & INN, BZD, KTMt \\
\hline Male & 60 & 26 & Right foot & Toe fractures & 6 & ANT, TMD, BZD, PGB \\
\hline Male & 78 & 69 & Left hand & $\begin{array}{l}\text { Amputation index } \\
\text { finger }\end{array}$ & 3 & BZD, PGB, TMD \\
\hline Female & 59 & 16 & Right hand & Carpal tunnel surgery & 4 & PGB, ANT \\
\hline Female & 42 & 10 & Right hip & Hip fracture & 4 & TMD, PGB, OXT \\
\hline Male & 37 & $4^{* *}$ & Left foot & $\begin{array}{l}\text { Ankle sprain and } \\
\text { fracture }\end{array}$ & 7 & KTM, PGB, FE \\
\hline $\begin{array}{l}\text { F: } 6 \\
\text { M: } 5\end{array}$ & $\begin{array}{l}53 \pm \\
10\end{array}$ & $37 \pm 26$ & $\begin{array}{l}\text { R: 3(U); 2(L) } \\
\text { L: 3(U); 3(L) }\end{array}$ & & $6.0 \pm 2.4$ & \\
\hline
\end{tabular}

Notes. ${ }^{* *}=$ less than six months; $\mathrm{Dx}=$ diagnosis. Sex: $\mathrm{F}=$ female; $\mathrm{M}=$ male. Affected limb: $\mathrm{U}=$ upper; $\mathrm{L}=$ lower. Pain medication: $\mathrm{ACE}=$ acetaminophen; $\mathrm{ANT}=$ antidepressive; $\mathrm{BZD}=$ benzodiazepine; $\mathrm{CAL}=$ calcitonin $; \mathrm{CBZ}=$ cyclobenzaprine, $\mathrm{CNZ}=$ clonazepam; $\mathrm{COD}=$ codeine $; \mathrm{FE}=$ fentanyl $; \mathrm{GBP}=$ gabapentin $; \mathrm{INN}=$ morphine; $\mathrm{KTM}(\mathrm{t})=$ ketamine $($ topic$) ; \mathrm{NAB}=$ nabilone; NSAID $=$ nonsteroidal anti-inflammatory drug; $\mathrm{OXT}=$ oxycodone; $\mathrm{PGB}=$ pregabalin; $\mathrm{TMD}=$ tramadol. 


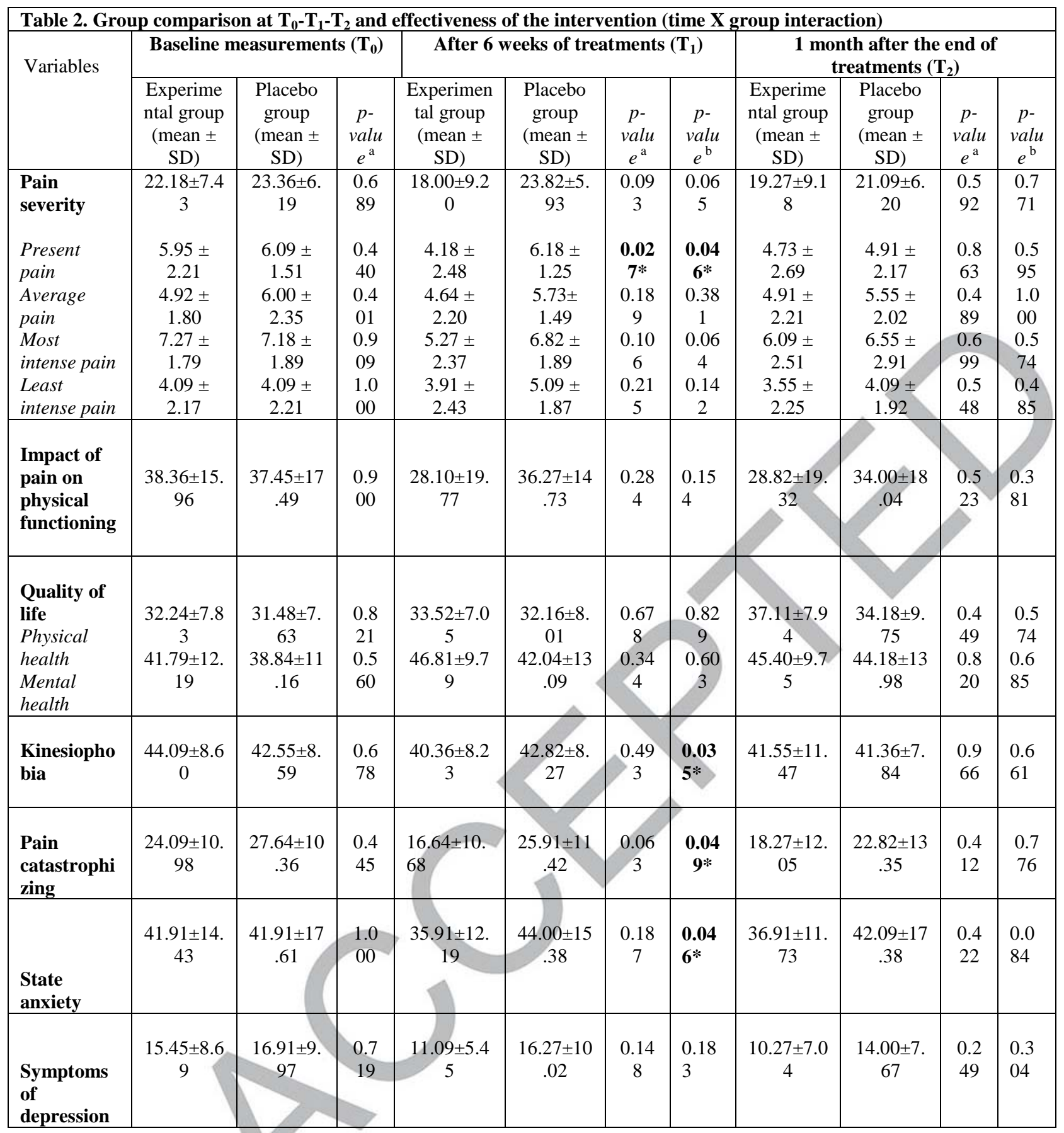

Notes. $* p<0,05 .{ }^{\mathrm{a}}$ Group comparison at a given measuring time; ${ }^{\mathrm{b}}$ Between group comparison (Group $\mathrm{x}$ Time interaction) 

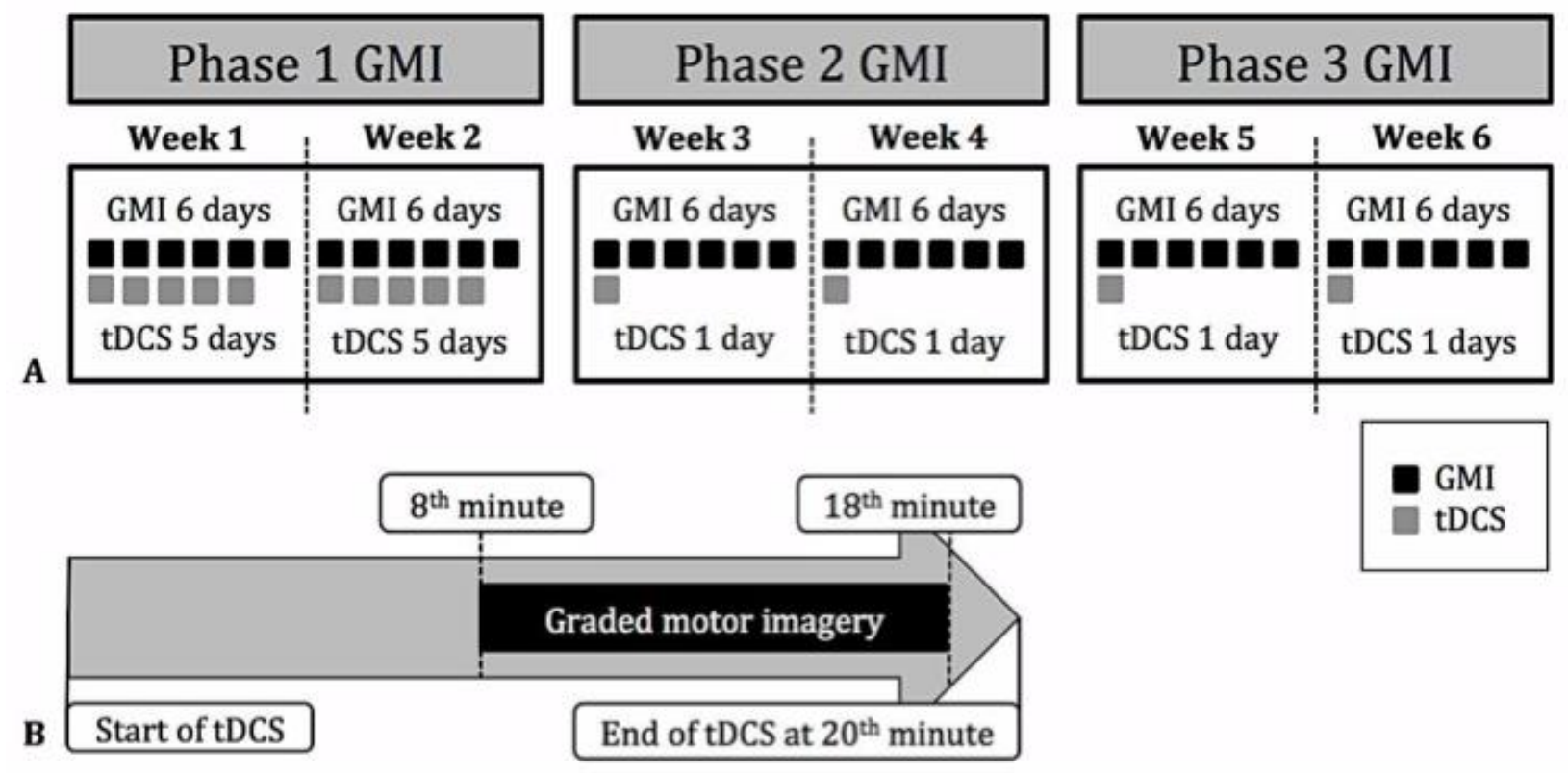

\section{Figure 1. Experimental design}

(a) Experimental design for each phase of GMI plus tDCS stimulation for the 6 weeks of treatment. (b) Timing of the application protocol of tDCS during GMI for the 14 treatments that occurred in the experimental settings. 


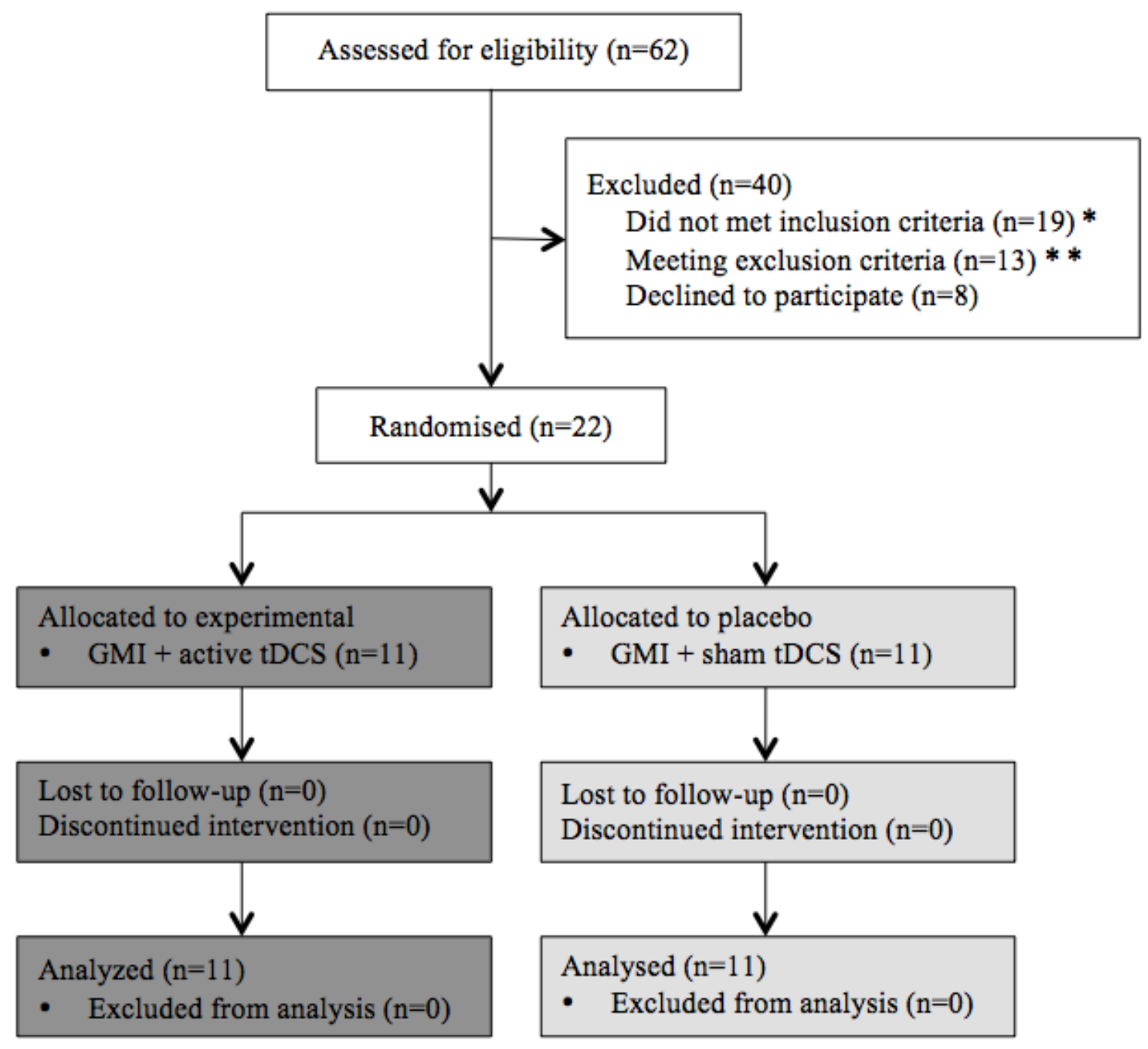

Figure 2. CONSORT flow chart

* $n=12$ with CRPS type 1 who did not fulfill recognized diagnostic criteria, $n=7$ with unstable medication; ** $\mathrm{n}=3$ with other chronic pain, $\mathrm{n}=1$ with unstable medical condition, $\mathrm{n}=2$ with head trauma, $\mathrm{n}=1$ with frequent severe headaches, $\mathrm{n}=2$ with epilepsy. GMI = Graded Motor Imagery; tDCS $=$ transcranial Direct Current Stimulation.

Copyright (C) 2017 Wolters Kluwer Health, Inc. Unauthorized reproduction of the article is prohibited. 


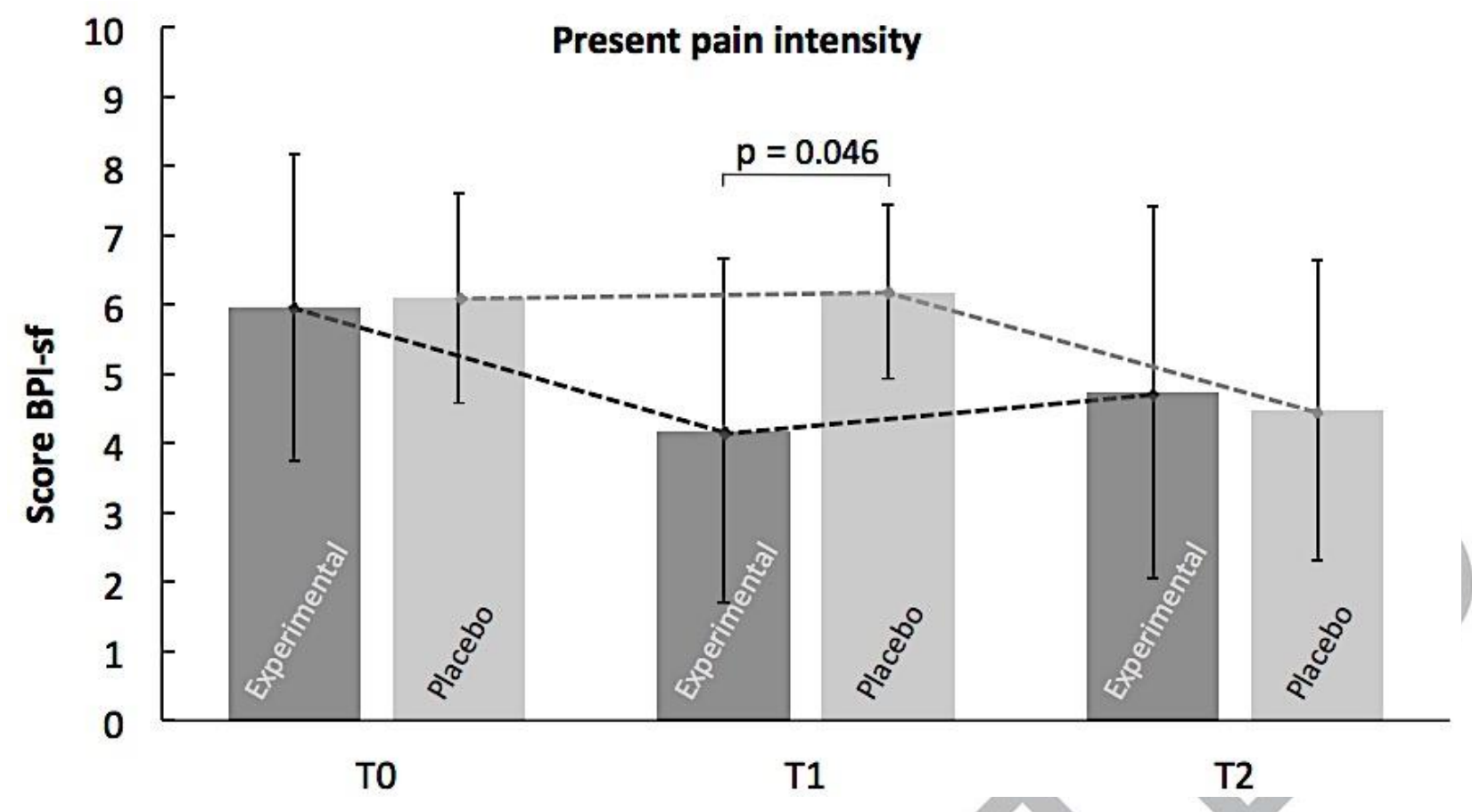

Figure 3. Progression of Present Pain intensity scores through time for both groups

Copyright (C) 2017 Wolters Kluwer Health, Inc. Unauthorized reproduction of the article is prohibited. 